\title{
Nanostructured Ti thin films by magnetron sputtering at oblique angles
}

\author{
R. Alvarez ${ }^{1, *}$, J.M. Garcia-Martin ${ }^{2}$, A. Garcia-Valenzuela ${ }^{1}$, M. Macias-Montero ${ }^{1}$, F.J. \\ Ferrer $^{3}$, J. Santiso ${ }^{4}$, V. Rico ${ }^{1}$, J. Cotrino ${ }^{1}$, A.R. Gonzalez-Elipe ${ }^{1}$, A. Palmero ${ }^{1}$ \\ ${ }^{1}$ Instituto de Ciencia de Materiales de Sevilla (CSIC-US), Americo Vespucio 49, 41092 \\ Seville, Spain \\ ${ }^{2}$ IMM- Instituto de Microelectrónica de Madrid (CNM-CSIC), Isaac Newton 8, 28760, \\ Tres Cantos, Madrid, Spain \\ ${ }^{3}$ Centro Nacional de Aceleradores (CSIC-US), Avda. Thomas A. Edison 7, 41092, \\ Sevilla (Spain) \\ ${ }^{4}$ Institut Català de Nanociència i Nanotecnologia, ICN2 (CSIC-Cerca). Campus de la \\ UAB, Edifici ICN2 08193 Bellaterra, Spain
}

\begin{abstract}
.-
The growth of Ti thin films by the magnetron sputtering technique at oblique angles and at room temperature is analysed from both experimental and theoretical points of view. Unlike other materials deposited in similar conditions, the nanostructure development of the Ti layers exhibits an anomalous behaviour when varying both the angle of incidence of the deposition flux and the deposition pressure. At low pressures, a sharp transition from compact to isolated, vertically aligned, nanocolumns is obtained when the angle of incidence surpasses a critical threshold. Remarkably, this transition also occurs when solely increasing the deposition pressure under certain conditions. By the characterization of the Ti layers, the realization of fundamental experiments and the use of a simple growth model, we demonstrate that surface mobilization processes associated to a highly directed momentum distribution and the relatively high kinetic energy of sputtered atoms are responsible for this behaviour.

* Email: rafael.alvarez@icmse.csic.es
\end{abstract}




\section{I.- Introduction}

The oblique angle configuration is a useful geometrical arrangement to promote the vacuum growth of nanostructured layers with high porosity and large specific surfaces [1]. The main feature of evaporation techniques operating under this configuration is the oblique arrival of sublimated species at a substrate and their subsequent condensation in the form of tilted columnar structures with diameters in the order of few tens nanometers [2]. This geometrical arrangement has also been tested with magnetron sputtering (MS) techniques [3-8] obtaining films with diverse columnar and porous structures and outstanding properties for applications in medicine, photovoltaics, microfluidics, catalysis or sensors, among others [1]. For example, in a recent work, we prepared by this technique operated at oblique angles (MS-OAD) a nanostructured coating made of $\mathrm{Ti}$ nanocolumns that exhibited opposite behavior towards cell or bacteria proliferation, a property that makes this film suitable for medical applications [9].

MS is a widespread growth method fully scalable for mass production and widely employed in research and industry [10]. Unlike electron beam evaporation method working in full vacuum conditions, MS-OAD utilizes a powered target placed in a reactor chamber that contains an inert gas (usually argon) at low pressures. Thanks to the propagation of an electromagnetic signal, this gas turns into plasma [11], resulting in the acceleration of positive ions towards the target and the sputtering of atoms with kinetic energies around $10 \mathrm{eV}$ in a preferential direction perpendicular to the target surface [10]. These species pass through the plasma and are deposited on a tilted substrate where they give rise to a nanostructured film. Therefore, in contrast with the evaporation technique, the MS-OAD introduces particular features that may strongly influence the growth of the films: i) the plasma species may interact with the film during the deposition [12], ii) some 
sputtered species may collide with heavy particles in the plasma and gradually loss their kinetic energy and preferential direction before being deposited (this phenomenon is called thermalization) [13], and iii) the kinetic energy of sputtered particles may be high enough to induce atomic mobilization processes at the film surface (dubbed hyperthermal processes) that may alter the film morphology [14-16].

In a recent work [17] we have analyzed the MS-OAD of Au thin films with the idea of expanding the well-known Thornton's Structure Zone Model (SZM) [18-19], incorporating the tilt angle of the substrate as an additional degree of freedom. In agreement with numerous experiments at low temperatures, we concluded that four generic nanostructures can develop depending on the plasma gas pressure and the tilt angle of the substrate $[8,17]$. Remarkably, the growth of these gold nanostructures could be accurately explained by just considering the collisional transport of sputtered particles in the plasma gas and their subsequent deposition, disregarding any hyperthermal mobilization process on the film surface. Moreover, the same model was found adequate to describe the MS-OAD of $\mathrm{TiO}_{2}$ thin films [20], thus suggesting that hyperthermal processes might not be efficient to produce noticeable changes in the final film nanostructures, at least in these two reported cases. However, the comparison between the nanostructures appearing in these $\mathrm{Au}$ or $\mathrm{TiO}_{2}$ films with those in the nanocolumnar Ti coating mentioned above [9], strongly suggests that additional mechanisms may play a relevant role in this latter case. In fact, while the porous gold nanostructure found at low pressures and high deposition angles consisted of densely packed columnar arrays tilted more than $50^{\circ}$ with respect to the vertical, the nanostructure of $\mathrm{Ti}$ coatings prepared under similar experimental conditions presented a well-spaced and almost perpendicular nanocolumnar structure. In the present work, we have carried out a systematic study on the growth of nanostructured $\mathrm{Ti}$ films by MS-OAD, exploring a wide range of 
experimental deposition conditions. Through the characterization of the obtained nanostructures, the analysis of fundamental experiments performed under selected conditions and the help of a simple growth model, the main processes governing the growth of these Ti layers have been identified and general insights gained that may likely be extended to understand the growth and nanostructure development of other materials.

\section{II.-Experimental conditions}

We have deposited a series of Ti thin films by MS-OAD at different pressures and deposition angles. A $5 \mathrm{~cm}$ diameter Ti target was employed with argon as sputter gas and a base pressure in the chamber in the mid $10^{-7} \mathrm{~Pa}$ range. A flat $1 \mathrm{~cm}^{2} \mathrm{Si}(100)$ substrate (with surface roughness below $0.5 \mathrm{~nm}$ ) cleaned in an ultrasonic bath, was placed at 0.22 $\mathrm{m}$ from the target and tilted at different angles with respect to its normal (see figure $\mathrm{S} 1$ ). The argon pressure, $p_{g}$, was set to $0.15,0.5,1$ and $1.5 \mathrm{~Pa}$, whereas the tilt angle of the substrate, $\sigma$, was set to $0^{\circ}, 45^{\circ}, 60^{\circ}, 70^{\circ}, 80^{\circ}$ and $85^{\circ}$ in the case of $p_{g}=0.15 \mathrm{~Pa}$, and $0^{\circ}$, $45^{\circ}, 60^{\circ}$ in the higher pressure cases. The DC electromagnetic generator was set at a constant power of $300 \mathrm{~W}$, conditions at which the visible plasma glow covers a volume extending up to $7 \mathrm{~cm}$ from the target and remains more than $15 \mathrm{~cm}$ away from the film. A cylindrical metallic chimney with $5 \mathrm{~cm}$ radius and $9 \mathrm{~cm}$ long was placed besides the target to collimate the ballistic flux of material and to trap the sputtered species that stay thermalized in the plasma phase (see ref. [21] for more details). The film temperature was always below $350 \mathrm{~K}$ during the sputtering process, whereas the deposition times ranged from 90 to 200 minutes.

Films were characterized by field emission scanning electron microscopy (FESEM) from two different perspectives, $\Delta$ and $\Pi$, defined in figure 1. Rutherford Backscattering 
Spectroscopy (RBS) was employed to assess the areal density of the films: experiments were carried out in the $3 \mathrm{MV}$ tandem accelerator of the National Center for Accelerators (Seville, Spain) with a beam of $1.5 \mathrm{MeV}$ alpha particles and a passivated implanted planar silicon (PIPS) detector located at $165^{\circ}$ scattering angle, with accumulated doses about 1.5 $\mu \mathrm{C}$, and $\sim 1 \mathrm{~mm}$ beam spot diameter. The RBS spectra were simulated with the SIMNRA code [22], whereas the density of each film was calculated by dividing the areal density by the thickness, as obtained from the cross-sectional FESEM image. Moreover, for comparison purposes, a Ti thin film was deposited by the electron beam-assisted physical vapour deposition at a zenithal deposition angle of $85^{\circ}$ using the experimental set-up described in reference [23].

Crystal quality and texture of the films were assessed by X-ray diffraction (XRD) in a Bragg-Brentano configuration. A XRD diffractometer with 4-angle goniometer (Bruker, D8 Advance) was used to explore the preferential orientation of the crystal domains in the films by means of pole figure analysis of different reflections in a wide range of tilt angles, $\chi=0-70^{\circ}$.

\section{III.-Growth Model}

Following the same approach than in previous works [17, 23, 24], our growth model selects the minimum set of processes that may explain the most outstanding nanostructural features observed in the films upon variation of experimentally controllable quantities. We consider the deposition of Ti atoms on a two-dimensional flat substrate that defines the $\mathrm{x}-\mathrm{y}$ plane of coordinates, whereas the $\mathrm{z}$ axis is defined by the direction perpendicular to it. The three dimensional space is divided into a $N_{L} \times N_{L} \times N_{H}$ cubic grid, where each cell has the value 1 if it contains a deposited $\mathrm{Ti}$ atom and 0 
otherwise. Each cell, with an estimated size equivalent to the typical atomic volume in the material $(\sim 0.4 \times 0.4 \times 0.4 \mathrm{~nm})$, represents an atom in the network. The Ti atoms are sputtered from the cathode with a momentum distribution $F(\vec{p})$, where $\vec{p}$ is the lineal momentum, and experience different scattering events in the plasma gas, arriving at the film with a momentum distribution function, $f(\vec{p})$. Then, the deposition process is described as follows: once the Ti atoms approach the film surface, they follow a straight trajectory with momentum $\vec{p}$ until they hit the surface, where the following hyperthermal processes are taken into account (see figure $2 \mathrm{a}$ and $2 \mathrm{~b}$ for a scheme):

Kinetic energy-induced mobility. This process is well documented in the literature and considers that upon deposition vapour atoms may transfer part of its kinetic energy and momentum to a surface atom, breaking the bonds and inducing a preferential mobility in the direction defined by the lineal momentum of the vapour atom [14-16, 25]. The dynamics of such collision is quite complex and, for simplicity reasons, we assume the following mechanism: if the energy involved in the collision, $\varepsilon=p^{2} / 2 M_{T i}\left(M_{T i}\right.$ is the mass of the Ti atom) is above certain energy threshold, $\varepsilon_{K}$, the target atom breaks its bonds and both atoms are allowed to relax to an available next neighbor position within a cone region aligned with the momentum of the incoming particle (see figure $2 a$ ).

Biased diffusion: If the kinetic-energy induced mobility process is inefficient, either because the energy involved is below $\varepsilon_{K}$ or because there are no free next neighbour sites available for the target atom to relax, we assume that the incident atom may keep part of its momentum in the direction parallel to the surface, $p_{\|}$, and slide over it until it becomes deposited by sticking at an obstacle site (see figure 2b). As stated in ref. [15-16, 26], this process takes place if the energy associated to that momentum, $p_{\|}^{2} / 2 M_{T i}$, is above a 
certain value, $\varepsilon_{\mathrm{BD}}$, and the angle of incidence above certain angular threshold with respect to the normal to the surface, $\theta_{\mathrm{BD}}$.

If none of the abovementioned processes takes place, the arriving atom is deposited just at the landing position. Consequently, the difference between this growth model and those previously employed in references $[17,20]$ is the addition of hyperthermal processes. In order to discuss the results, we specifically make use of the concept of thermalization degree of sputtered particles, usually named $\Xi$, which is a non-dimensional quantity deduced in ref. [21] for the MS-OAD of Ti thin films as $\Xi \simeq 13.2 \times p_{g} L \mathrm{~Pa}^{-1} \mathrm{~m}^{-1}$, with $L$ the distance between the target and the film. The value of $\Xi$ accounts for the thermalization degree at the substrate position of sputtered particles by interaction with the plasma gas: when $\Xi<<1$ the deposition flux is highly energetic and directional, whereas $\Xi>>1$ implies that particles are thermalized and possess low energy and no preferential directionality.

The model is solved by knowing $F(\vec{p}), f(\vec{p}), \varepsilon_{K}, \varepsilon_{B D}$ and $\theta_{B D} \cdot F(\vec{p})$ has been calculated by using the SRIM code [27], through which the function $f(\vec{p})$ was obtained using the well-known and tested SIMTRA code [28-29]. The value of $\varepsilon_{K}$ was taken as the heat of sublimation of $\mathrm{Ti}, \varepsilon_{K}=4.9 \mathrm{eV}$, whereas the biased diffusion energy and angular thresholds have been estimated using molecular dynamics simulations and the Kalypso code [30]. By this calculation, we have thrown numerous Ti atoms with different energies and angles onto a Ti flat surface and found the values $\varepsilon_{B D} \sim 1 \mathrm{eV}$ and $\theta_{B D} \sim 30^{\circ}$ , which are quite similar to those reported in ref. [26] for $\mathrm{Cu}$. 
It is worth noticing that other physical interactions and processes involved in the thin film growth have not been included in the model. This is the case of thermally-activated mobility processes that, due to the low value of the film temperature during growth $\sim 0.15 \times T_{m}\left(T_{m}\right.$ is the melting film temperature), can be reasonably discarded. This coincides with the deductions derived from the well-known structure zone model [31], in the sense that surface shadowing dominates over thermally activated processes when the growth temperature remains below $0.3 \times T_{m}$. In addition, we have not introduced resputtering processes because they play a minor role in the absence of negative ion species [32]. Argon ion bombardment effects are also neglected because of two reasons: i) the plasma glow is separated from the film more than $15 \mathrm{~cm}$ and, ii) the Ar plasma is maintained by a DC electromagnetic signal, implying that plasma ions possess low kinetic energy when arriving at the film surface [20,33].

To prove the reliability of the model, we have calculated the density of the simulated films. In general, these possess three different regions as a function of height: a first accommodation layer $\sim 10 \mathrm{~nm}$ thick close to the substrate, a second bulk region where the nanostructure is clearly defined, and a third region corresponding to the film surface. When the film is thick enough, the bulk region represents by far the largest part of the film, and thus, the overall density would tend to the bulk value. For this reason, in our calculations we have taken the local density in the bulk region as equivalent to that of the whole film. i.e., the number of occupied cells in one slice of material at a given height in the bulk divided by the number of cells in the slice.

\section{IV.-Experimental Results}


The nanostructural and crystallographic features of the films have been analysed as a function of the tilt angle of the substrate, $\sigma$, for a given thermalization degree of sputtered particles, $\Xi$. A series of FESEM images along the $\Delta$ and $\Pi$ directions are presented in figures 3 and 4, respectively, for $\Xi=0.4 \quad\left(p_{g}=0.15 \mathrm{~Pa}\right)$ and increasing values of $\sigma$. There, it is clear that the layers deposited for $\sigma=0^{\circ}, 45^{\circ}$ and $60^{\circ}$ depict rather compact structures, whereas for $\sigma=70^{\circ}$ a columnar morphology is formed made of vertically aligned and well-separated columns with diameters ranging from 50 to $100 \mathrm{~nm}$. Remarkably, for $\sigma=80^{\circ}$ and $\sigma=85^{\circ}$, the structure is rather similar, being the columns almost vertically aligned in both cases, a quite different behaviour to that reported for $\mathrm{Au}$ or $\mathrm{TiO}_{2}$ thin films prepared by MS-OAD at low pressures. In these latter cases, the tilting degree of the columnar structures progressively increased with $\sigma$, up to $\sim 50^{\circ}$ with respect to the vertical when $\sigma=85^{\circ}[17,20]$. Another significant difference in the present case of $\mathrm{Ti}$ is the remarkable abrupt transition from compact to columnar morphologies when the deposition angle varies between $\sigma=60^{\circ}$ and $\sigma=70^{\circ}$, a phenomenon we dub columnar breakdown. This behaviour is quite different from that observed for $\mathrm{Au}$ or $\mathrm{TiO}_{2}$ thin films, where a smooth shift from compact to tilted nanocolumnar structures took place for angles between $\sigma=60^{\circ}$ and $\sigma=80^{\circ}$ [17]. Interestingly, the columnar breakdown involves a sharp variation of films' density as reported in Figure 5a where we plot the relative density of the Ti films, in comparison with a fully dense layer, as a function of $\sigma$. There, it is clear that, with a density above $80 \%$, films are quite compact for $\sigma<70^{\circ}$ whereas the abrupt change in morphology for $\sigma=70^{\circ}$ translates into a noticeable density drop that reaches a value of $30 \%$ when $\sigma=85^{\circ}$.

Changes in morphology and density are accompanied by significant modifications in the films crystallographic structure. The XRD diagrams in Figure 6a show that all the Ti films 
are crystalline, although the relative intensities of the (100), (002) and (101) diffraction peaks of the hcp structure vary significantly with the deposition angle. This change in the peaks intensities clearly points to a certain texture evolution: the high intensity XRD peaks for the (002) and (100) reflections in the film deposited at $\sigma=0^{\circ}$ indicates a mixed a/c preferential orientation, a situation that evolves to an almost pure $c$-axis orientation for the films deposited at $\sigma=45^{\circ}$. Above this deposition angle, the samples show much weaker diffraction peaks suggesting a loss of preferential orientation, as well as a lower crystal quality (see figure 6a). This transition is in perfect correspondence with the morphology and density changes associated to the transition from compact morphology for $\sigma \leq 60^{\circ}$ to a highly porous microstructure when $\sigma>60^{\circ}$. To further assess the evolution of thin film texture, polar plots have been obtained to study the preferential orientation of crystal domains as a function of deposition angle. Figure $6 \mathrm{~b}$ shows the pole figures for the (002) reflections in the films deposited at different angles. The areas with maximum intensity correspond to the stereographic projection of the orientation distribution of $c$-axis oriented domains. Films grown with $\sigma=0^{\circ}, 45^{\circ}$ and $60^{\circ}$ show that (002) reflections approach the zenith position $\chi=0^{\circ}$ indicating a $c$-axis orientation very close to the vertical direction. The enlargement of the region around $\chi=0^{\circ}$ (in the insets) reveals a slight tilt of the $c$-axis of about $\chi=2^{\circ}$ and $2.5^{\circ}$ away from the vertical direction towards the incident flux of material for the $\sigma=45^{\circ}$ and $60^{\circ}$ samples, respectively. Films grown at $\sigma=70^{\circ}, 80^{\circ}$ and $85^{\circ}$ still reveal a certain preferential orientation of crystallites, although with a very broad distribution of c-axis orientations and a progressive tilt from $\chi=35^{\circ}$, to $45^{\circ}$ and $50^{\circ}$, respectively.

Films deposited at a higher pressure of $0.5 \mathrm{~Pa}(\Xi=1.45)$ show rather compact structures for tilt angles between $\sigma=0^{\circ}$ and $\sigma=45^{\circ}$ (see figures 7 and 8) and the evolution of a 
clearly defined columnar structure for $\sigma=60^{\circ}$. This result contrasts with the rather compact microstructure obtained when $\sigma=60^{\circ}$ and $\Xi=0.4$ (figures 3 and 4), which suggest that, remarkably, the sole increase of the argon gas pressure has induced a columnar breakdown for $\sigma=60^{\circ}$. This behaviour is again quite different to that reported for $\mathrm{Au}$ or $\mathrm{TiO}_{2}$, where the lower directionality of the deposition particles due to the increase of the argon pressure leads to less defined columnar microstructures [17]. We will come back to this point when discussing the results of the growth model.

Increasing the pressure also induces changes in the film density (see figure $5 b$ ), with values around $80 \%$ and $70 \%$, for $\Xi=1.45$ and $\sigma=0^{\circ}$ and $\sigma=45^{\circ}$, respectively. An abrupt drop to a density below $50 \%$ in the $\sigma=60^{\circ}$ case confirms the columnar breakdown taking place under those conditions. Titanium thin films deposited at even higher thermalization degrees, $\Xi=2.9\left(p_{g}=1 P a\right)$ and $\Xi=4.3\left(p_{g}=1.5 P a\right)$ (images shown in the supplementary information file S2) possess a density almost independent of $\sigma$, in all cases around $50 \%$ of that of a compact material (see figure $5 \mathrm{~b}$ ). This result suggests that sputtered particles have lost their preferential directionality and arrive at the film surface according to an isotropic angular distribution function, independent of $\sigma$.

\section{VI.- Growth simulation and Discussion}

The results presented in the previous section indicate that the nanostructural development of Ti thin films deposited by MS-OAD is rather different from that of $\mathrm{Au}$ or $\mathrm{TiO}_{2}$ deposited in similar conditions. This suggest that additional fundamental processes, besides the collisional transport of sputtered particles through the plasma gas and the surface shadowing mechanism, must be at play during the growth of the former. In particular, we propose that hyperthermal processes may explain the nanostructural transitions reported above, and that the well-tested model to describing the growth of $\mathrm{Au}$ 
and $\mathrm{TiO}_{2}$ films must be extended accordingly. We will show that, by comparing the solutions of the model and the experimental results, we are able to draw relevant conclusions on the growth of Ti by MS-OAD.

Low pressure cases $\left(\Xi=0.4, p_{g}=0.15 P a\right)$

The growth model has been solved under different conditions for $N_{L}=2000$ and different values of $N_{H}$, so that the simulated thin films possess the same thickness than the experimental ones. Simulations for $\Xi=0.4\left(p_{g}=0.15 P a\right)$ and different values of $\sigma$ are presented besides the experimental FESEM images in figures 3 and 4, evidencing the existence of a good agreement in all studied cases, except for $\sigma=70^{\circ}$ which will be discussed later. Primarily, for $\sigma=0^{\circ}$ and $\sigma=45^{\circ}$, the obtained compact structures reflect the low efficiency of surface shadowing effects at these low incidence angles [17] and the relatively high energy that sputtered particles carry prior to their deposition, a situation that favours the mobilisation of surface atoms by hyperthermal processes. Figure 3 and 4 also shows that the rather compact structures simulated for $\sigma=60^{\circ}$ and $\sigma=70^{\circ}$ evolve into a vertically oriented columnar nanostructure for $\sigma=80^{\circ}$ and $85^{\circ}$. To understand the columnar breakdown and explain how the vertical columns are formed, we show in Figure 9 the magnitude of the average displacements per deposited atom caused by hyperthermal processes as a function of $\sigma$, differentiating those that take place either vertically or horizontally with respect to the substrate. This figure shows that, for low incident angles, the arrival of energetic atoms mainly causes downwards displacements, likely contributing to densify the films and remove any emerging pattern. However, when $\sigma>70^{\circ}$, displacements are mainly horizontal in the $\Delta$ direction due to both, the glancing incidence of sputtered species and the appearance of new surfaces (the side of the columns), in a process we dub dragging mechanism. In fact, the dragging 
mechanism strongly influences the columnar growth: equivalent simulations in Figure 10 in the absence of hyperthermal processes show that nanocolumns would naturally tilt towards the sputtered flux (see figure 10a), i.e., in the direction opposite to $\Delta$, as it was previously reported for $\mathrm{Au}$ and $\mathrm{TiO}_{2}$ thin films. Therefore, and regarding the almost vertical alignment of the columns in the Ti cases, it seems that when $\sigma>70^{\circ}$, the dragging mechanism is responsible for mobilizing surface atoms in the $\Delta$ direction, compensating the natural tilt of the columns and straightening them up. This is illustrated in figure 11 , where we show how the dragging mechanism affects the otherwise tilted columnar growth, straightening the columns up, and making them cast a larger shadow over the substrate. This makes columns grow more separated, promoting the growth of films with lower densities.

The dragging mechanism does not only explain why columns remain almost vertical no matter the value of $\sigma$, but also the sharp columnar breakdown found at low pressures. In fact, and according to classical growing concepts accounting for the appearance of tilted nanocolumns in evaporated thin films at glancing angles [1], their formation is promoted by the appearance of slightly taller surface nuclei in the first stages of growth that become larger and turn into columns thanks to surface shadowing mechanisms. Our model indicates that this picture still holds for MS-OAD, although the incorporation of the dragging mechanism contributes to straighten these nuclei up as soon as they emerge and to enhance the surface shadowing processes thanks to their larger projected shadow. All these factors combined are responsible for promoting the growth of vertically aligned and very spaced columns, as well as for a sharp transition between compact and columnar morphologies. 
Experimental evidence on the existence of the dragging mechanism can be concluded by comparing the reported nanostructures in figures 3 and 4 with that of a $\mathrm{Ti}$ thin film deposited by the electron beam-assisted evaporation technique at low temperatures (details on the deposition method appear in refs. [23, 34]), where the low kinetic energy of evaporated species when arriving at the film surface $(\sim 0.1-0.2 \mathrm{eV})$ should preclude any dragging mechanism. In a remarkably good agreement with the simulations in absence of hyperthermal processes (Figure 10a), the cross-sectional FESEM image of this film (in Figure 10b) shows that the Ti nanostructures appear tilted towards the vapour flux. This result clearly demonstrates that the dragging mechanism, and thus the hyperthermal processes, have a direct influence on the formation of vertically aligned columns.

As mentioned before, the simulations for $\sigma=70^{\circ}$ and $\Xi=0.4$ do not match well with experiments in figures 3 and 4: while a columnar arrangement is experimentally obtained, the model predicts a rather compact structure. We think that this disagreement is caused by the sharpness of the transition and the simplified vision of the hyperthermal mechanisms employed in our model, which renders a threshold angle for the columnar breakdown of $\sigma \sim 75^{\circ}$, instead of the experimental value $\sim 70^{\circ}$. In any case, the good agreement between simulations and experiments can be quantitatively confirmed by comparing measured and calculated film densities in figure $4 \mathrm{a}$, which again shows a good concordance. Unfortunately, no predictive assessment can be extracted from the model on the film texture evolution (c.f., Figure 6), since no crystalline planes can be made out from simulations. Yet, it seems quite remarkable that the texture of the films experiences a drastic modification for $\sigma>60^{\circ}$, i.e., when the dragging mechanism emerges as a dominant nanostructuring process, suggesting a possible link between both. However, the extension of this possible relation to other materials or conditions has to be taken 


\section{Film morphology at higher pressures}

For higher thermalization degrees, e.g., $\Xi=1.45\left(p_{g}=0.5 \mathrm{~Pa}\right)$, the results of the simulations also show a good agreement with experiments in figures 7 and 8 . For $\sigma=0^{\circ}$ and $\sigma=45^{\circ}$, simulations render compact films, whereas for $\sigma=60^{\circ}$ the structure evolves into vertically aligned columns. As mentioned before, this result is puzzling because it indicates that the sole increase of pressure when $\sigma=60^{\circ}$ promotes a columnar breakdown. According to our model, this phenomenon can be explained by considering the plot in Figure 12 that shows the proportion of arriving Ti atoms that may induce hyperthermal processes at the film surface, i.e. with kinetic energies above $\varepsilon_{K}$, for increasing thermalization degrees (pressures). As expected, the decreasing trend obtained indicates that the more thermalized sputtered atoms become, the less kinetic energy they carry, and hence less hyperthermal processes are induced. Therefore, a plausible explanation for this pressure-induced transition is as follows: the higher amount of energetic Ti atoms at low pressures is responsible for inducing numerous hyperthermal processes at the film surface which, at low deposition angles $\left(\sigma \leq 60^{\circ}\right)$, implies the removal of any emerging pattern and the promotion of a compact thin film growth. At higher pressures, on the other hand, the higher thermalization degree leads to a lower amount of energetic atoms, so that the occurrence of less hyperthermal processes allows 
for the formation of columns. In order to corroborate this result, in figure 13 we show the simulated structure for $\Xi=0.4\left(p_{g}=0.15 \mathrm{~Pa}\right.$, low pressure $)$ and $\sigma=60^{\circ}$ in the absence of hyperthermal processes, where a clear columnar nanostructure is obtained. All this confirms that the actual compact structure found at $60^{\circ}$ and low pressure (c.f. figures 3 and 4 for $\sigma=60^{\circ}$ ) is associated to highly efficient hyperthermal mobilization processes, and that the reported pressure-induced columnar breakdown is mediated by the different efficiency of hyperthermal processes during the film growth.

The conclusions above can be further validated by comparing the calculated and measured densities of the films for increasing pressures and different values of $\sigma$. Indeed, our model does not only reproduce the film density for $\Xi=1.45\left(p_{g}=0.5 \mathrm{~Pa}\right)$ for different values of $\sigma$, but also in the cases with $\Xi=2.9\left(p_{g}=1 P a\right)$ and $\Xi=4.3\left(p_{g}=1.5 P a\right)$, as depicted in figure 5b. In agreement with figure 12, when $\Xi=2.9\left(p_{g}=1 P a\right)$ and $\Xi=4.3\left(p_{g}=1.5 P a\right)$, the very high thermalization degree of sputtered atoms causes an important drop of kinetic energy that inhibits hyperthermal processes. This fact together with the loss of preferential directionality of sputtered species when arriving at the substrate explains why the film density presents a very weak dependence on pressure and substrate tilt angle in these cases.

All the simulations presented in this paper were performed on flat surfaces due to the 0.5 $\mathrm{nm}$ roughness of the Si substrates experimentally used. According to our experience, the final film morphology would be the same whenever the roughness of the substrate is kept low enough. For instance, we have used medical grade Ti6Al4V substrates of $2 \mathrm{~mm}$ thickness (mechanically polished to a mirror finish), with a roughness of about $3 \mathrm{~nm}$ over a $4 \mu \mathrm{m}^{2}$ area, to grow the Ti nanostructures at low pressures and with $80^{\circ}$ tilt angle [9]. 
In this case, the obtained nanocolumnar morphology is the same as that grown on the $\mathrm{Si}$ substrates (depicted in figures 3 and 4). However, it is worth mentioning that if substrates were seeded, different nanostructures could be developed depending on the particular seed pattern and size. Indeed, these seeds could induce additional surface shadowing mechanisms and surface correlations that would promote the appearance of different structures. In this paper, due to the complexity and vast number of conditions associated to the presence of seeds, we have focussed on the nanostructuration process in simple conditions, i.e., on almost flat or low roughness substrates, demonstrating the importance of hyperthermal processes in the formation of Ti nanocolumns by MS-OA. The model presented here reproduces the nanostructural features of the $\mathrm{Ti}$ thin films grown at different pressures and tilt angles of the substrate and explains the sharp columnar breakdown that takes place when increasing the tilt angle at low pressures, and when increasing the deposition pressure for $\sigma=60^{\circ}$. Moreover, the good match between experimental and calculated film densities ensures the accuracy of our model to predict film densities and porosities.

As a final remark, it is worth mentioning the relevance of developing a growth model such as the one presented in this paper. It allows the computational analysis of the film growth in different conditions and geometries, providing a first assessment on the outcome of a particular experiment in a matter of hours (typical running time in an average personal computer of a $100 \mathrm{~nm} \times 100 \mathrm{~nm} \times 100 \mathrm{~nm}$ simulation is less than 1 hour). It can be employed, for instance, to give insights on the geometrical constraints to scale up the MS-OAD technique to industrial reactors and homogeneously coat large substrates. As mentioned in the introduction, in ref. [9] we demonstrated that the $\sigma=80^{\circ}$ case in figures 3 and 4, possesses a selective behavior when exposed to osteoblast cells and bacteria (allowing the growth of the former, and inhibiting the proliferation of the 
latter) due to its particular nanostructure. These phenomena are of the utmost importance for biomedical applications, since nanostructured coatings onto actual implants may significantly reduce the infection rate. Such application would require not only coating large surfaces (up to tens of $\mathrm{cm}^{2}$ ) but also, in some cases, using strategies to deposit with oblique incidence onto curved substrates. Moreover, the MS-OAD technique has also proven adequate for the development of devices in small scales for numerous applications, e.g., sensors, microfluidics, solar cells, plasmonics, etc. [1], for which the development of growth methods on large substrates is required. This issue represents a clear scientific and engineering challenge that demands the development of models like the one presented here.

\section{VII.- Conclusions}

In this paper we have studied the fundamentals of the growth of Ti thin films by magnetron sputtering at oblique angles. For this purpose, we have explored a wide range of deposition conditions and characterized the obtained Ti nanostructures as a function of the deposition angle and working pressures. The substantial differences found between the $\mathrm{Ti}$ nanostructures and those obtained for $\mathrm{Au}$ and $\mathrm{TiO}_{2}$ grown in similar conditions indicate that processes other than the collisional transport of sputtered species in the plasma and surface shadowing mechanism must be at play. To account for the different observations, we have obtained that kinetic energy-induced hyperthermal processes have a relevant influence on the development of the Ti nanostructures: a simple growth model accounting for these mechanisms has been developed that qualitatively explains the main morphological features of the Ti thin films as well as the nanostructural changes observed when varying the experimental conditions. Moreover, a quantitative agreement has been also found when comparing the density of the experimental and simulated layers. 


\section{Acknowledgements}

The authors thank Fundación Domingo Martínez, the Junta de Andalucía (P12-FQM2265) and the Ministry of Science and Innovation (Projects CONSOLIDER CSD200800023, MAT2013-42900-P, MAT2013-40852-R, MAT2014-59772-C2-1, MAT201129081) for financial support. 


\section{REFERENCES}

[1] Barranco A, Borras A, Gonzalez-Elipe A R, Palmero A 2015 Perspectives on Oblique Angle Deposition of Thin Films: From Fundamentals to Devices, Progress in Materials Science, doi: http://dx.doi.org/10.1016/j.pmatsci.2015.06.003

[2] Hawkeye M M, Taschuk M T, Brett M J 2014 Glancing Angle Deposition of Thin Films: Engineering the Nanoscale ISBN: 978-1-118-84756-5 John Wiley and Sons, Inc

[3] Zhou C M, Gall D 2006 Branched Ta nanocolumns grown by glancing angle deposition Appl. Phys. Lett. 88203117.

[4] Kesapragada S V, Gall D 2006 Anisotropic broadening of Cu nanorods during glancing angle deposition Appl. Phys. Lett. 89, 203121.

[5] Toledano D, Galindo R E, Yuste M, Albella J M, Sanchez O 2013

Compositional and structural properties of nanostructured $\mathrm{ZnO}$ thin films grown by oblique angle reactive sputtering deposition: effect on the refractive index $J$. Phys. D: Appl. Phys. 46, 045306.

[6] Ruffino F, Grimaldi M G 2012 Control of the Kinetic Roughening in Nanostructured Ag Films by Oblique Sputter-Depositions Nanosci. Nanotechnol. Lett. 4, 309.

[7] Patzig C, Karabacak T, Fuhrmann B, Rauschenbach B 2008 Glancing angle sputter deposited nanostructures on rotating substrates: Experiments and simulations J. Appl. Phys. 104, 094318.

[8] García-Martín J M, Alvarez R, Romero-Gómez P, Cebollada A, Palmero A 2010 Tilt angle control of nanocolumns grown by glancing angle sputtering at variable argon pressures Appl. Phys. Lett. 97,173103

[9] Izquierdo-Barba I, Arcos D, Alvarez R, Palmero A, García-Martin J M, Esteban J, Pérez-Jorge C, Vallet-Regí M 2015 Nanocolumnar coatings with selective behavior towards osteoblast and Staphylococcus aureus proliferation Acta Biomaterialia 15, 20.

[10] Reactive Sputter Deposition, Springer Series in Materials Science, edited by D Depla and S Mahieu, ISBN 978-3-540-76662-9, Springer-Verlag Berlin Heidelberg 2008. 
[11] Palmero A, Rudolph H, Habraken F H P M 2007 Characterization of a lowpressure argon plasma using optical emission spectroscopy and a global model $J$. Appl. Phys. 101, 053306.

[12] van Hattum E D, Palmero A, Arnoldbik W M, Rudolph H, Habraken F H P M 2007 On the ion and neutral atom bombardment of the growth surface in magnetron plasma sputter deposition Appl. Phys. Lett. 91, 171501

[13] Palmero A, Rudolph H, Habraken F H P M 2006 Generalized Keller-Simmons formula for nonisothermal plasma-assisted sputtering depositions Appl. Phys. Lett. 89, 211501.

[14] Smy T, Salahuddin M, Dew S K, Brett M J 1995 Explanation of spurious features in tungsten deposition using an atomic momentum model J. Appl. Phys. 78(6), 4157.

[15] Zhou X W , Wadley H N G 1999 Hyperthermal vapor deposition of copper: athermal and biased diffusion effects Surface Science 431, 42-57.

[16] Zhou X W , Wadley H N G 1999 Hyperthermal vapor deposition of copper: reflection and resputtering effects Surface Science 431, 58-73.

[17] Alvarez R, Garcia-Martin J M, Macias-Montero M, Gonzalez-Garcia L, Gonzalez J C, Rico V, Perlich J, Cotrino J, Gonzalez-Elipe A R, Palmero A 2013 Growth regimes of porous gold thin films deposited by magnetron sputtering at oblique incidence: from compact to columnar microstructures Nanotechnology 24, 045604.

[18] Thornton J A 1974 Influence of apparatus geometry and deposition conditions on the structure and topography of thick sputtered coatings J. Vac. Sci. Technol. 11, 666.

[19] Thornton J A 1975 Influence of substrate temperature and deposition rate on structure of thick sputtered Cu coatings J. Vac. Sci. Technol. 12, 830

[20] Alvarez R, Lopez-Santos C, Ferrer F J, Rico V, Cotrino J, Gonzalez-Elipe A R, Palmero A 2015 Modulating Low Energy Ion Plasma Fluxes for the Growth of Nanoporous Thin Films Plasma Processes and Polymers 12, 719.

[21] Alvarez R, Garcia-Martin J M, Lopez-Santos M C, Rico V, Ferrer F J, Cotrino J, Gonzalez-Elipe A R, Palmero A 2014 On the Deposition Rates of Magnetron Sputtered Thin Films at Oblique Angles Plasma Processes and Polymers 11, 571. 
[22] Mayer M 1997 SIMNRA User's Guide Tech Rep IPP 9/113, Max-Plank-Institut fur Plasmaphysik, Garching, Germany.

[23] Alvarez R, Gonzalez-Garcia L, Romero-Gomez P, Rico V, Cotrino J, GonzalezElipe A R, Palmero A 2011 Theoretical and experimental characterization of TiO2 thin films deposited at oblique angles J. Phys. D: Appl. Phys. 44, 385302.

[24] Alvarez R, Romero-Gomez P, Gil-Rostra J, Cotrino J, Yubero F, Palmero A, Gonzalez-Elipe A R 2010 On the microstructure of thin films grown by an isotropically directed deposition flux J. Appl. Phys. 108, 064316

[25] Torre J D, Gilmer G H, Windt D L, Kalyanaraman R, Baumann F H, O’Sullivan P L, Sapjeta J, Rubia T D, Rouhani M D 2003 Microstructure of thin tantalum films sputtered onto inclined substrates: Experiments and atomistic simulations J. Appl. Phys. 94(1), 263.

[26] Kools J C S 2005 Suppression of nanoscopic shadowing during physical vapor deposition by biased diffusion J. Vac. Sci. Technol. A 23(1), 85-89.

[27] http://www.srim.org/

[28] van Aeken K, SIMTRA available at www.draft.ugent.be/

[29] van Aeken K, Mahieu S, Depla D 2008 The metal flux from a rotating cylindrical magnetron: a Monte Carlo simulation J. Phys. D: Appl. Phys. 41, 20530.

[30] Karolewski M A 2005 Kalypso: A software package for molecular dynamics simulation of atomic collisions at surfaces Nucl. Instr. Meth. B 230, 402-405. Software available at https://sites.google.com/site/kalypsosimulation/

[31] Mukherjee S, Gall D 2013 Structure zone model for extreme shadowing conditions Thin Solid Films 527, 158-163.

[32] Song Q, Wu B, Xie B, Huang F, Li M, Wang H, Jiang Y, Song Y 2009 Resputtering of zinc oxide films prepared by radical assisted sputtering J. Appl. Phys. 105, 044509.

[33] Welzel T, Ellmer K 2013 Comparison of ion energies and fluxes at the substrate during magnetron sputtering of $\mathrm{ZnO}: \mathrm{Al}$ for DC and RF discharges J. Phys. D: Appl. Phys. 46(31), 315202.

[34] Alvarez R, Lopez-Santos C, Parra-Barranco J, Rico V, Barranco A, Cotrino J, Gonzalez-Elipe A R, Palmero A 2014 Nanocolumnar growth of thin films 
1

2

3

4

5

6

7

8

9

10

11

12

13

14

15

16

17

18

19

20

21

22

23

24

25

26

27

28

29

30

31

32

33

34

35

36

37

38

39

40

41

42

43

44

45

46

47

48

49

50

51

52

53

54

55

56

57

58

59

60

deposited at oblique angles: Beyond the tangent rule J. Vac. Sci. Technol. B 32(4), 041802.

[35] Sanjeev S K, Kim D Y 2013 Abnormal residual stress in nanostructured Al thin films grown on Ti/glass substrates Current Applied Physics 13, 1874

[36] Sharma S K, Kim M-S, Kim D Y, Yu J-S 2013 Al nanorod thin films as anode electrode for Li ion rechargeable batteries Electrochimica Acta 87, 872 


\section{Figure Caption}

Figure 1.- Definition of the $\Pi$ and $\Delta$ directions

Figure 2.- Hyperthermal processes considered in the model. a) Kinetic energy-induced mobility, b) biased diffusion.

Figure 3.- View from the $\Pi$ direction of the obtained nanostructures for the Ti thin films for $\Xi=0.4\left(p_{g}=0.15 P a\right)$, and different tilt angles of the substrate. The FESEM images of the films are displayed in the left column, whereas in the right column the corresponding results of the simulations are presented.

Figure 4.- View from the $\Delta$ direction of the same Ti thin films presented in figure 3 . The FESEM images of the films are displayed in the left column, whereas in the right column the corresponding results of the simulations are presented.

Figure 5.- Experimental density of the films as a function of the substrate angle, together with the results of the simulations. a) $\Xi=0.4\left(p_{g}=0.15 P a\right)$, and b) $\Xi=1.45$ ( $\left.p_{g}=0.5 P a\right), \Xi=2.9\left(p_{g}=1 P a\right)$ and $\Xi=4.3\left(p_{g}=1.5 P a\right)$.

Figure 6. a) XRD peaks of the films grown at $\Xi=0.4\left(p_{g}=0.15 P a\right)$, for different values of the substrate angle. b) Pole figures of the 002 reflection for the films grown for $\Xi=0.4$ $\left(p_{g}=0.15 P a\right)$. The tilt angle $\chi$ varies from 0 to $90^{\circ}$ and the rings correspond to steps of $10^{\circ}$. The insets correspond to close up of the $\chi=0-10^{\circ}$ region. 
Figure 7.- View of the obtained nanostructures for the Ti thin films in the $\Pi$ direction for $\Xi=1.45\left(p_{g}=0.5 P a\right)$, and different tilt angles of the substrate. The FESEM images of the films are displayed in the left column, whereas in the right column the corresponding results of the simulations are presented.

Figure 8.- View of the obtained nanostructures for the Ti thin films in the $\Delta$ direction for $\Xi=1.45\left(p_{g}=0.5 P a\right)$, and different tilt angles of the substrate. The FESEM images of the films are displayed in the left column, whereas in the right column the corresponding results of the simulations are presented.

Figure 9. Vertical and horizontal lateral mobility processes caused by hyperthermal processes per deposited particle, for $\Xi=0.4\left(p_{g}=0.15 P a\right)$ and different substrate angles.

Figure 10.- a) Result of the model in absence of hyperthermal processes for $\Xi=0.4$ and $\sigma=85^{\circ}$, b) FESEM image of a Ti thin film grown by evaporation at an oblique angle of $85^{\circ}$ for the sake of comparison.

Figure 11.- Scheme on the influence of the dragging mechanism in the columnar growth.

Figure 12.- Calculated proportion of $\mathrm{Ti}$ atoms with kinetic energy above $\varepsilon_{K}$ when arriving at the substrate as a function of the thermalization degree.

Figure 13.- Image of a simulated film in the conditions $\Xi=0.4$ and $\sigma=60^{\circ}$ when hyperthermal processes are inhibited. 
Figure 1

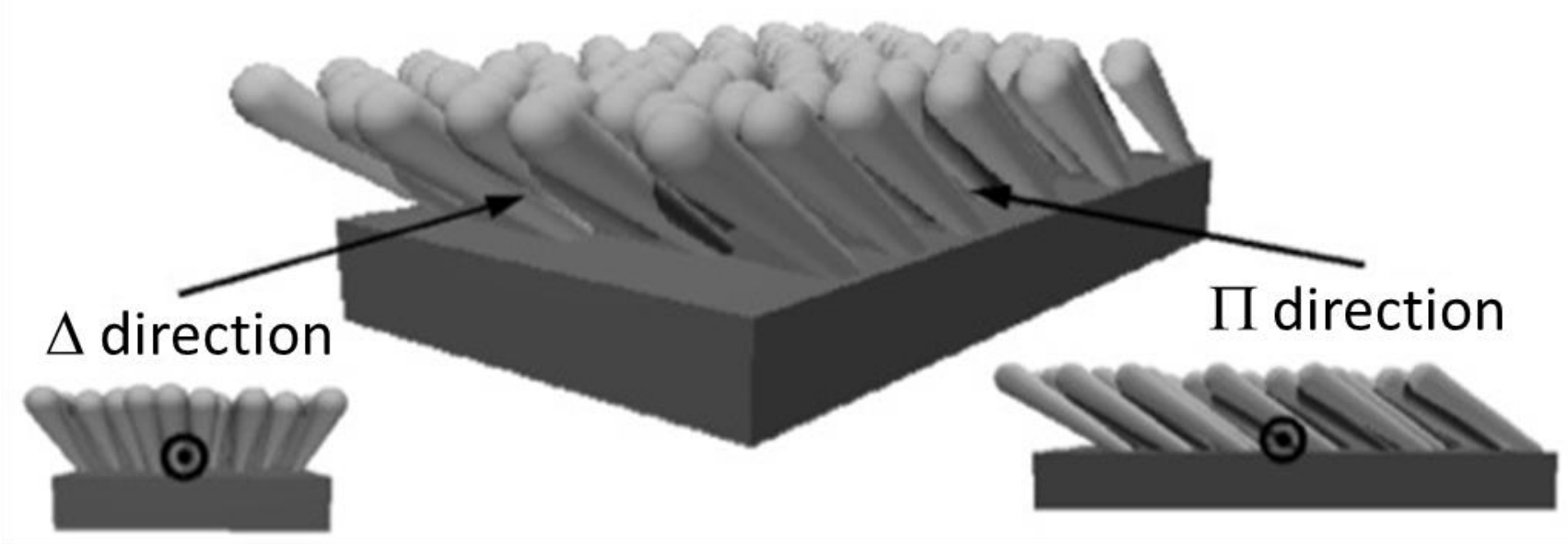


Figure 2
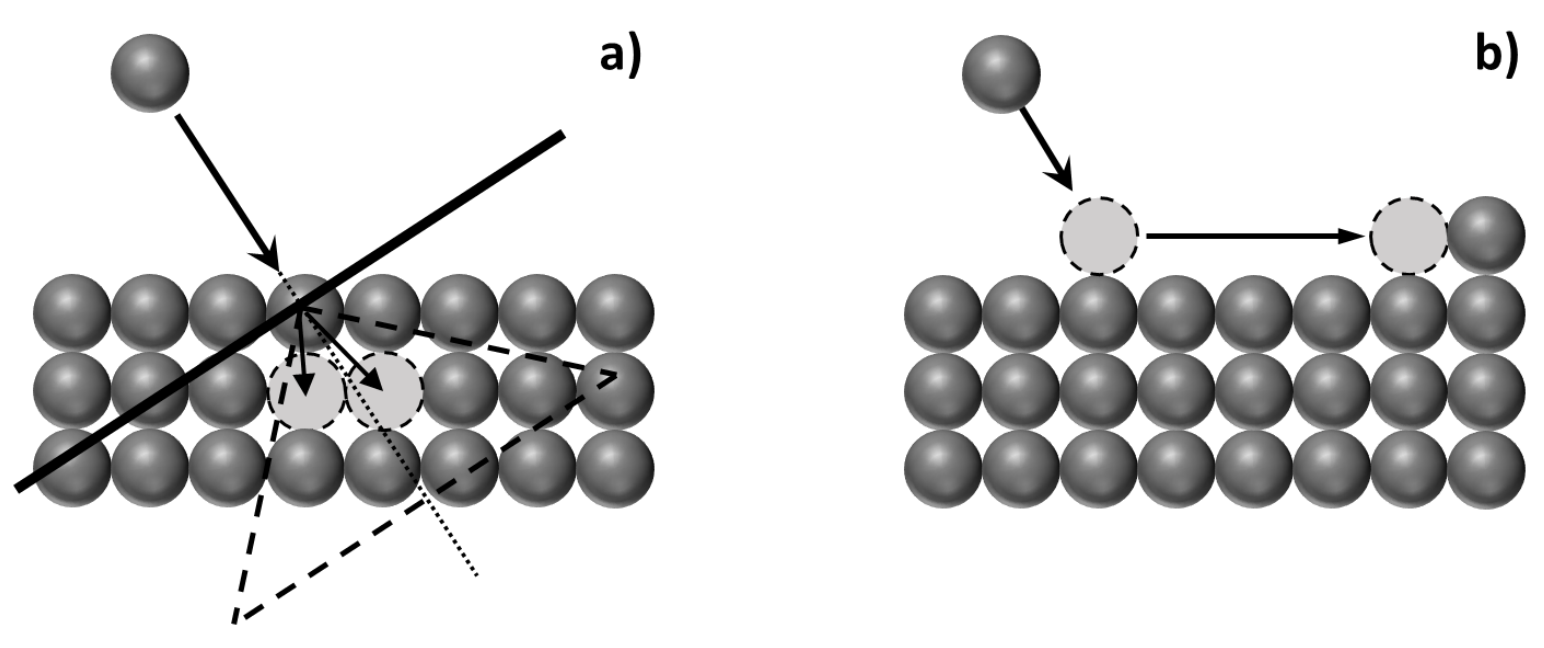
Figure 3
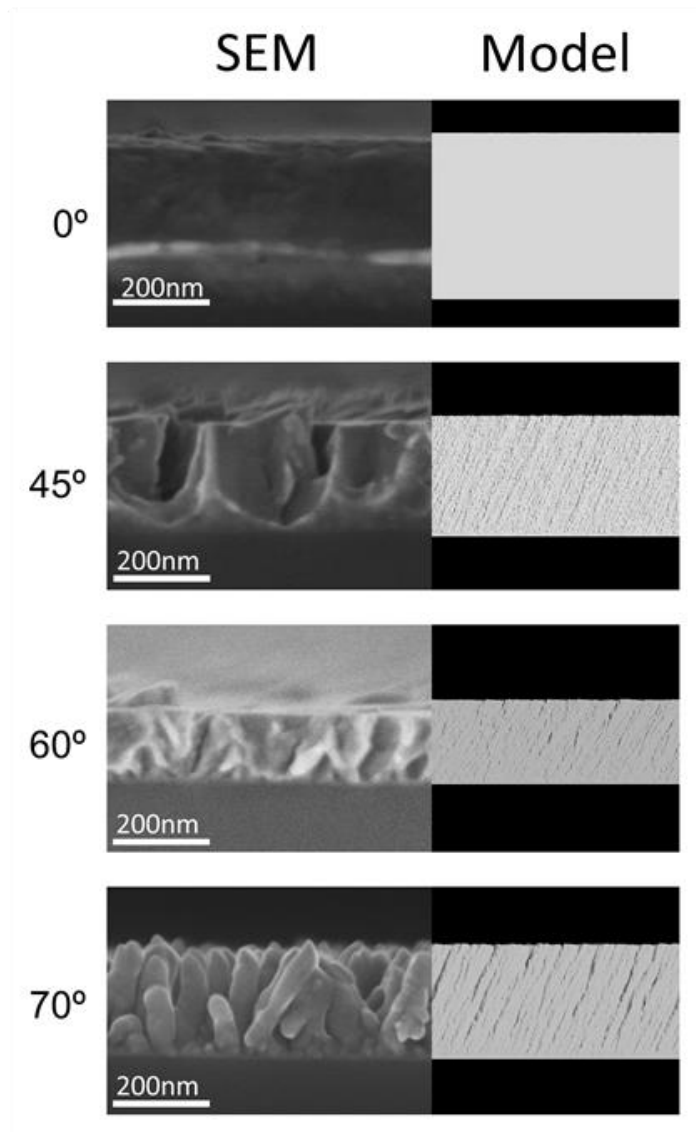

$80^{\circ}$
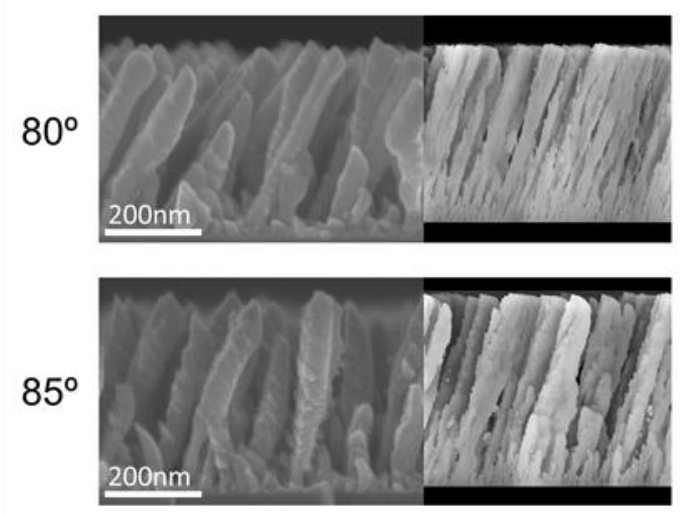

$\Delta$ direction

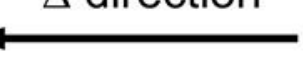


Figure 4
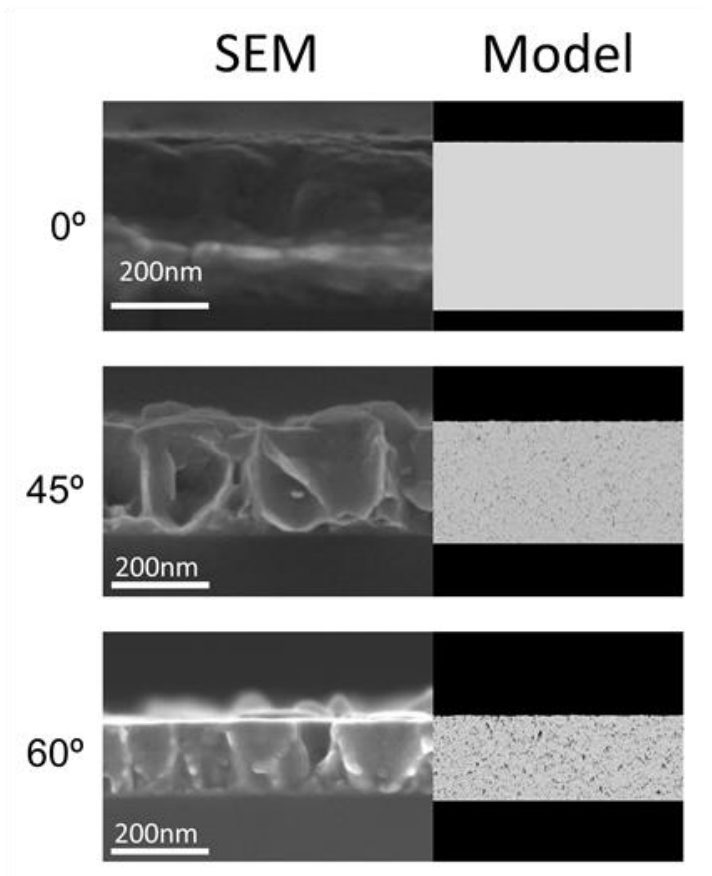

$70^{\circ}$

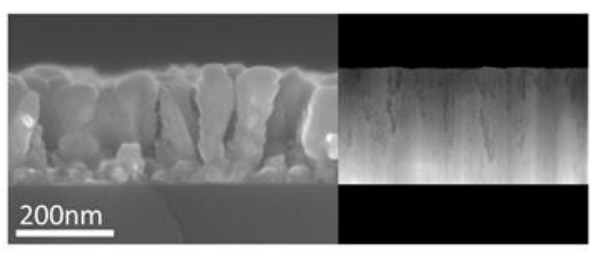

$80^{\circ}$

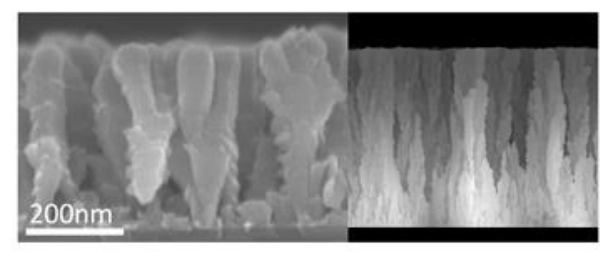

$85^{\circ}$

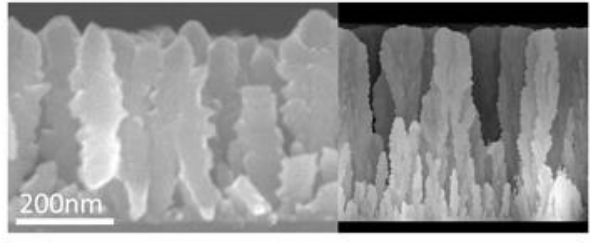

$\Pi$ direction 
Figure 5
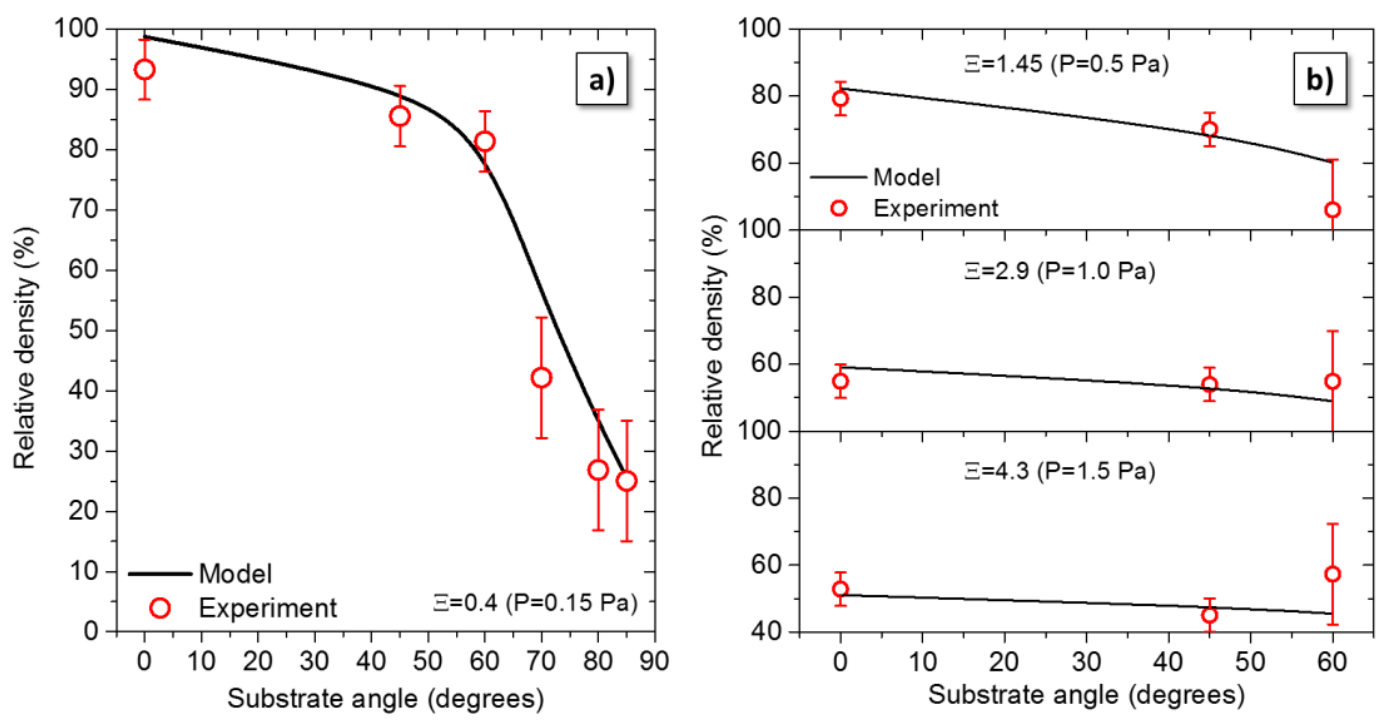
Figure 6

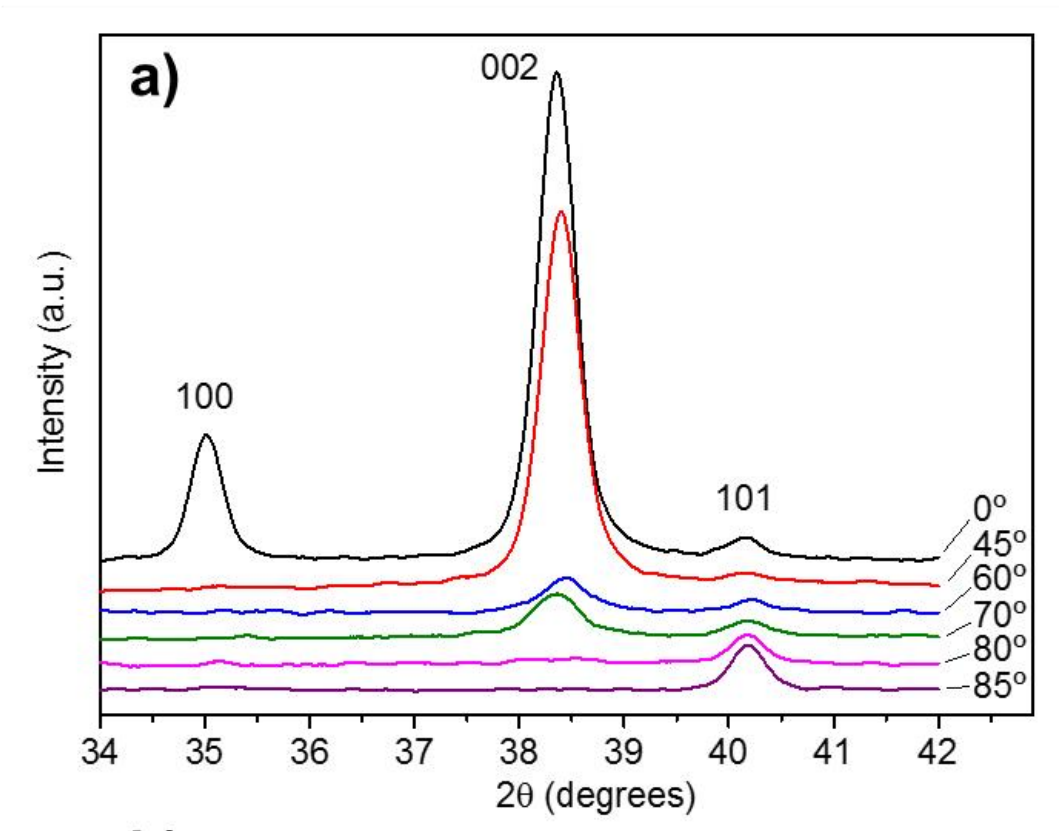

b)
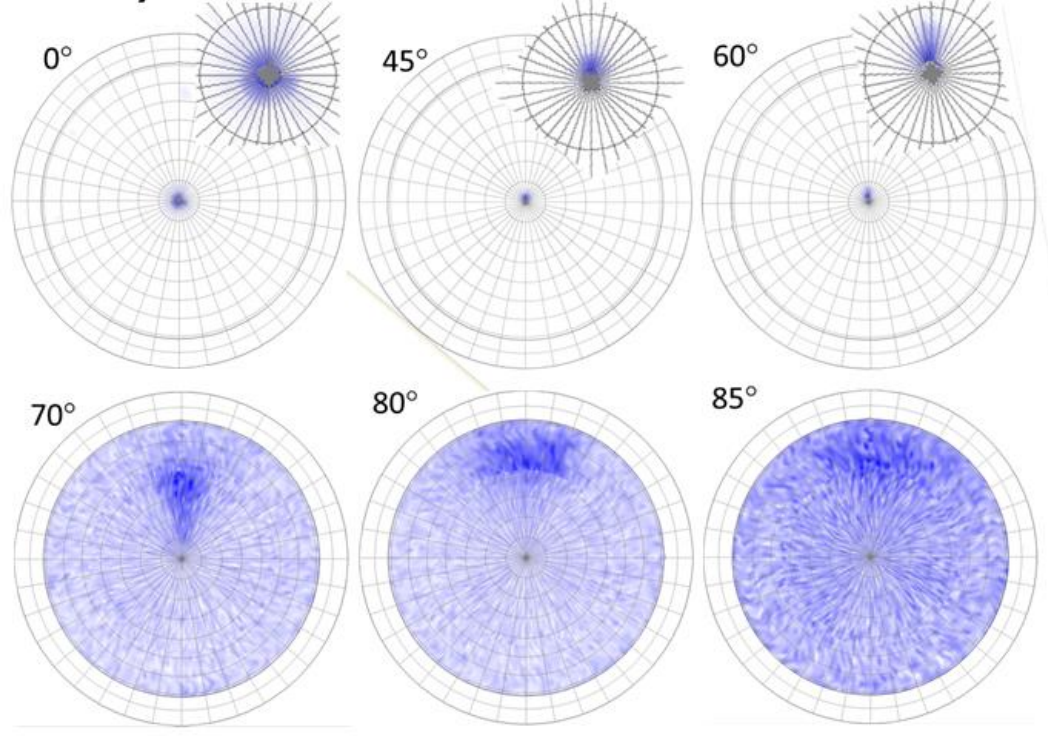

$85^{\circ}$ 


\section{Figure 7}

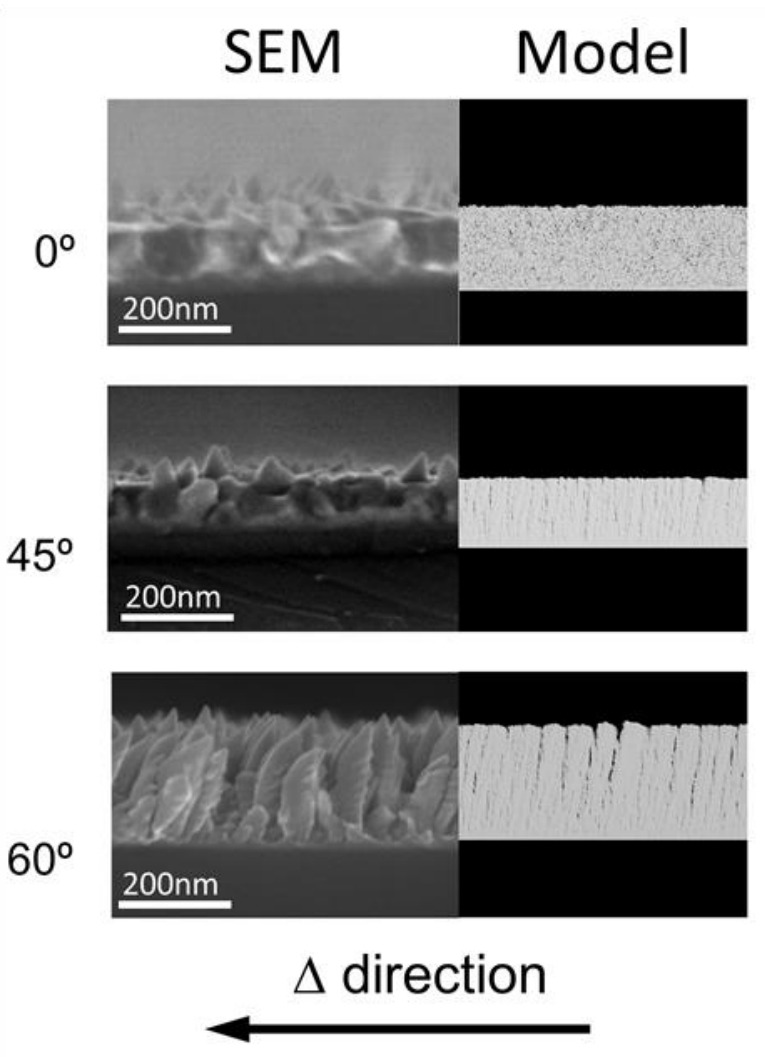


Figure 8

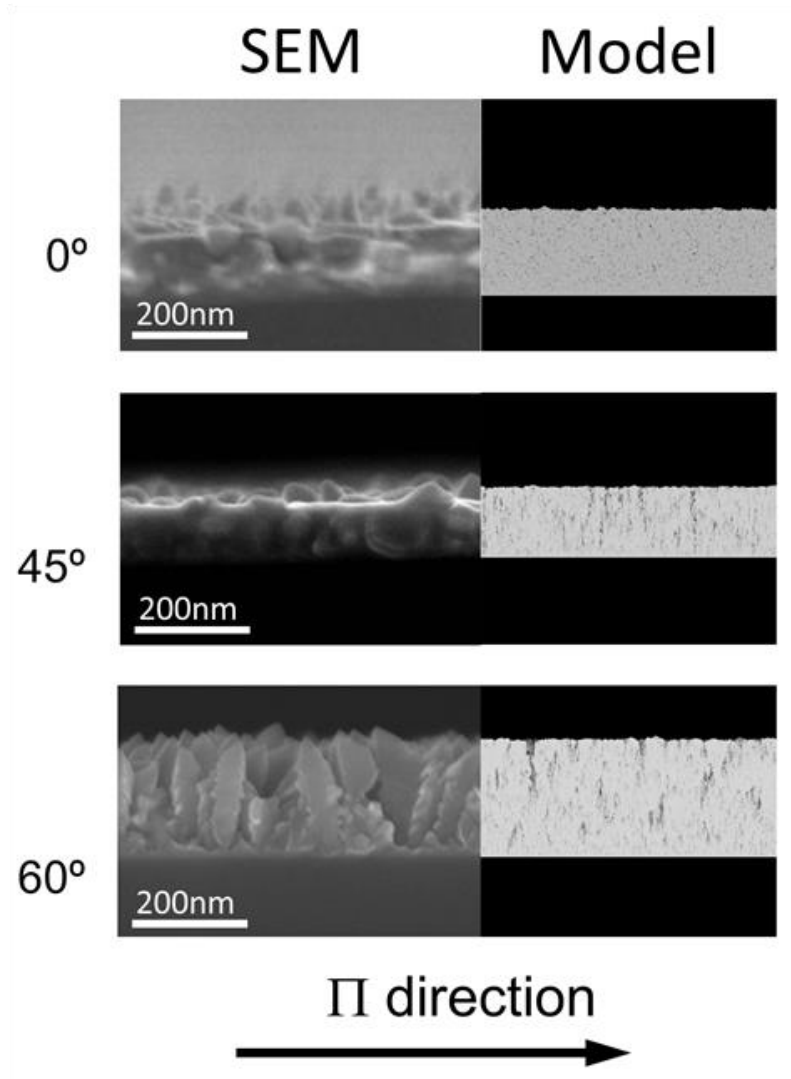


Figure 9

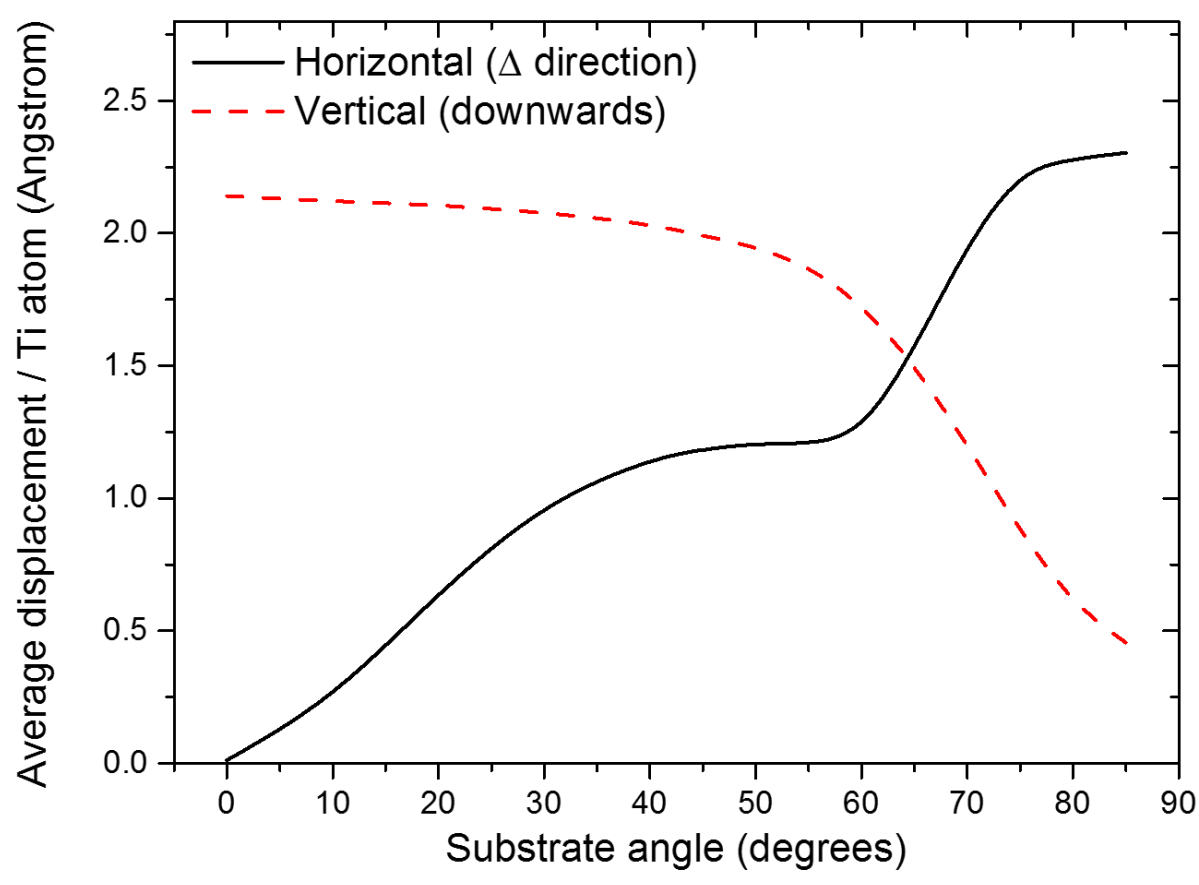


Figure 10
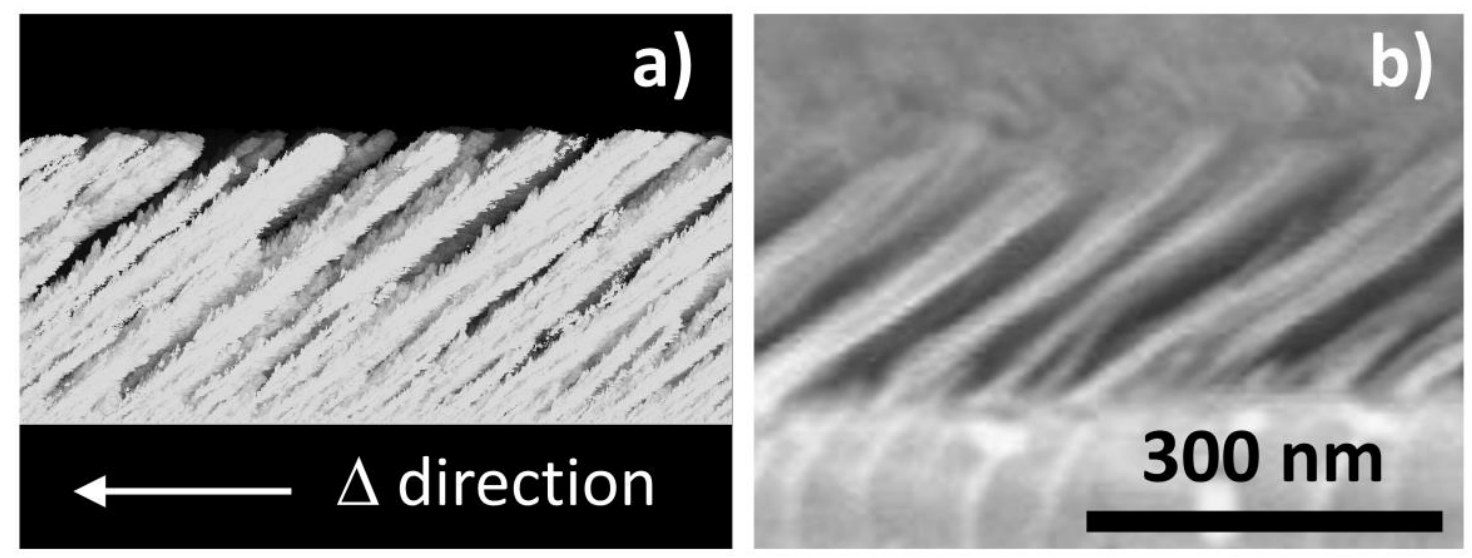
Figure 11

Sputtered atom
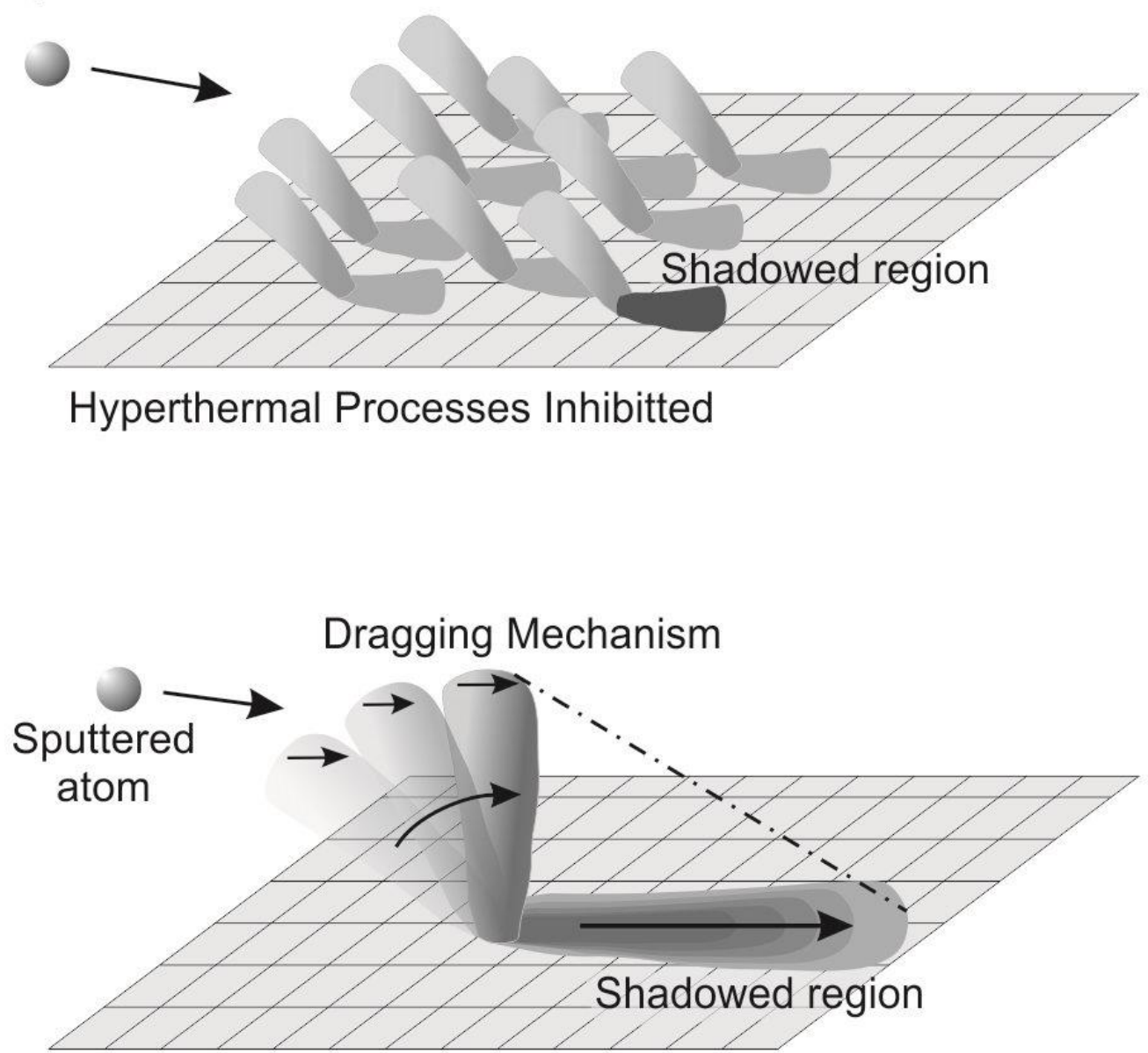
Figure 12

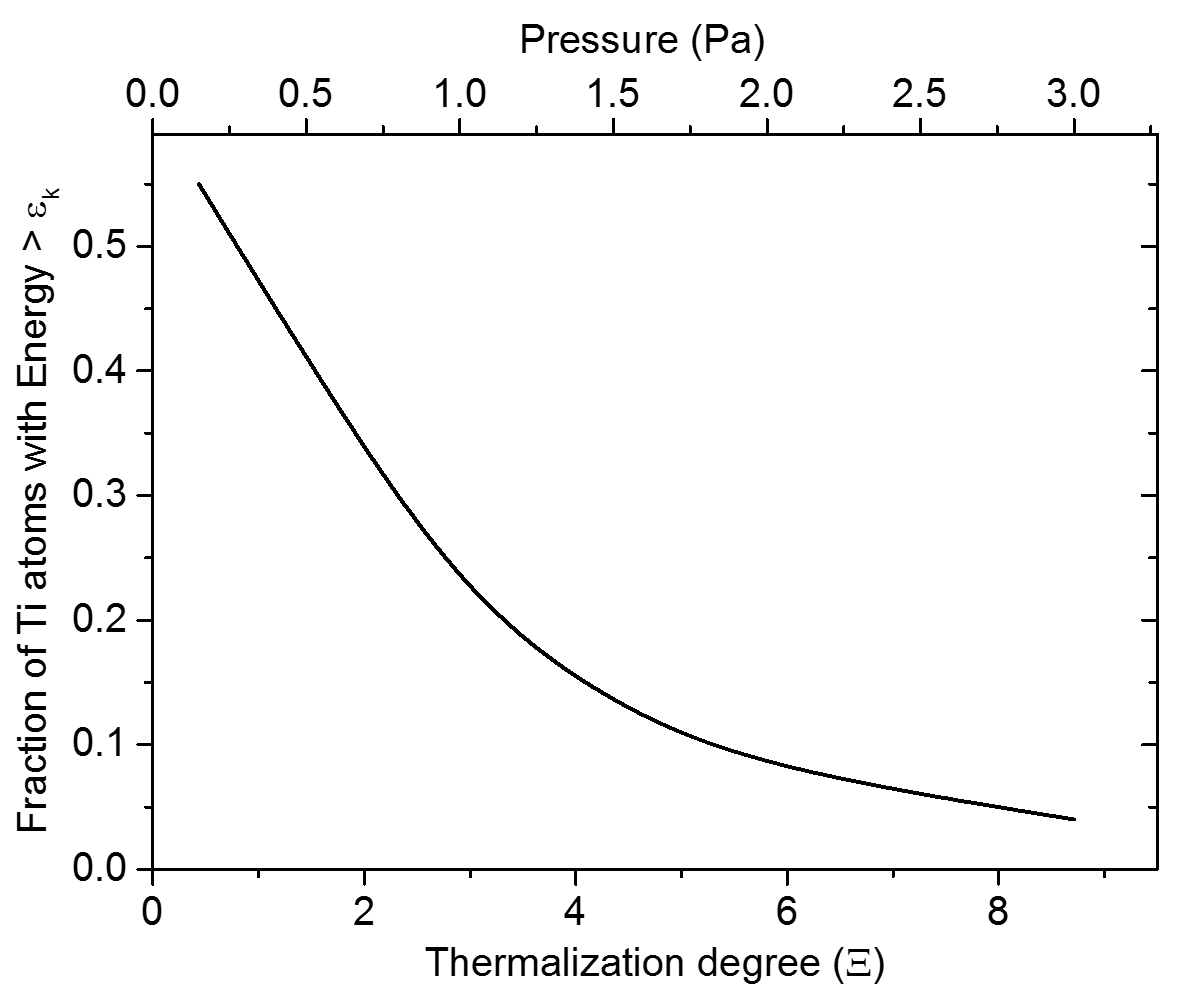


Figure 13

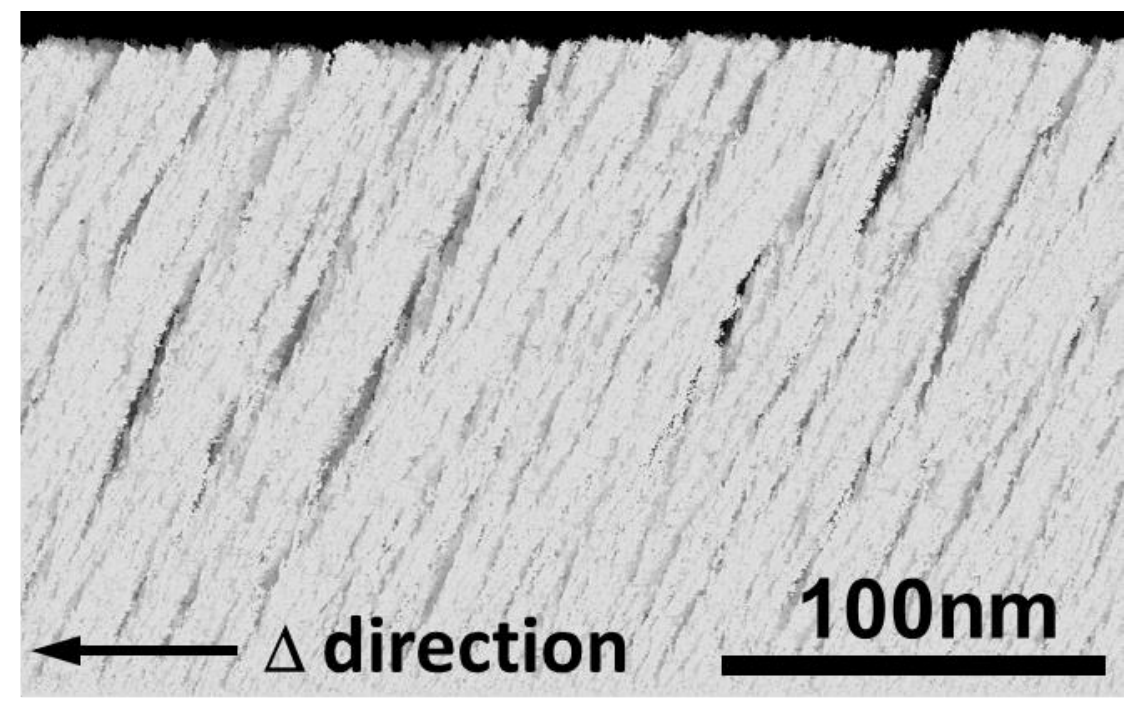




\section{Supplementary information}

Figure S1: Experimental Setup

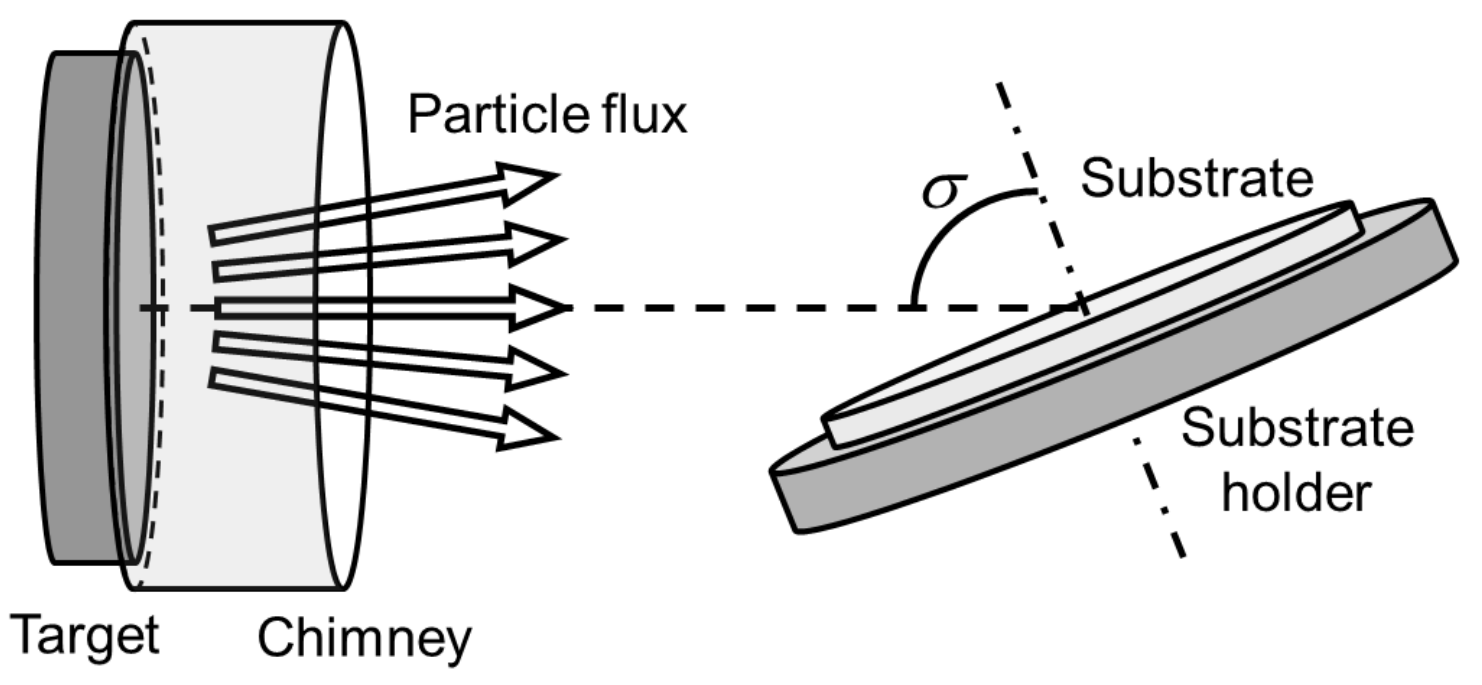


Figure S2: FESEM images of the Ti thin films grown at $0^{\circ}, 45^{\circ}$ and $60^{\circ}$, at higher thermalization degrees: $\Xi=2.9\left(p_{g}=1 \mathrm{~Pa}\right)$ and $\Xi=4.3\left(p_{g}=1.5 \mathrm{~Pa}\right)$.

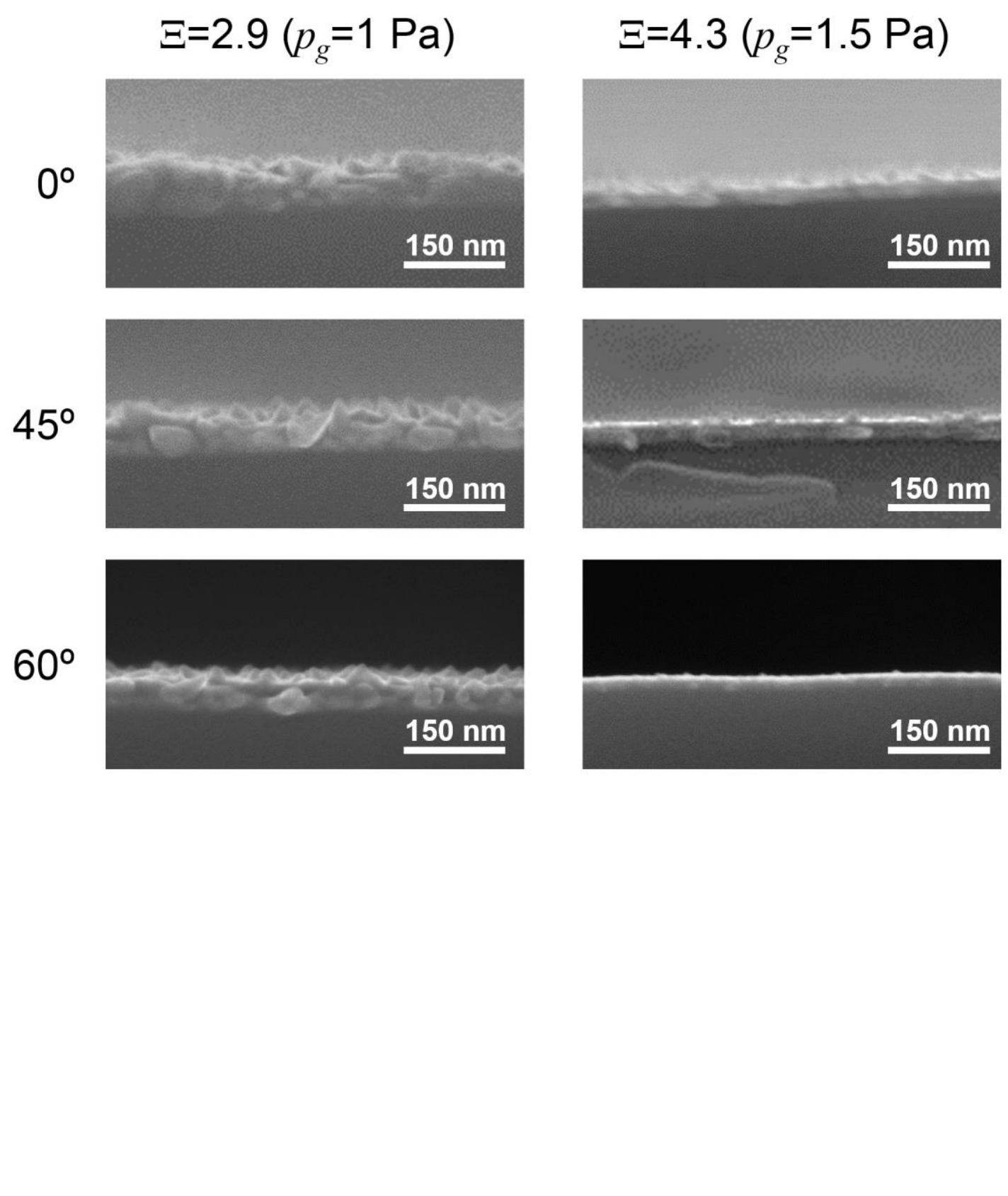

